\title{
The Use of Chipless Sensors with RFID for Condition Monitoring
}

\author{
H. Gokay Bilic, Tarik Buyukoztekin, Serhan Ozdemir \\ Izmir Institute of Technology \\ Department of Mechanical Engineering \\ Artificial Iytelligence \& Design Laboratory \\ Izmir, Turkey \\ gokaybilic@iyte.edu.tr, tarikbuyukoztekin@iyte.edu.tr, serhanozdemir@iyte.edu.tr
}

\begin{abstract}
This paper presents the development phases and overview of developing research in the area of RFID condition monitoring, focusing on chipless sensors especially use in strain and temperature sensing applications. Classification of RFID sensors and smart material fundamentals are reviewed. The compact and feasible design of RFID sensors will be considered, as well as with the effect of different material usage. Finally, the use of chipless sensors with different condition monitoring applications and their challenges are investigated.
\end{abstract}

Index Terms-chipless, sensor, tag, RFID, condition monitoring, strain.

\section{INTRODUCTION}

Radio Frequency Identification (RFID) technology is an emerging part of our lives such as gated communities, collecting tolls, inventory control, logistics, indoor positioning, condition monitoring and fault detection applications etc. RFID systems contain two components, the reader also known as interrogator, and the transponder also known as tag. The reader is the component that activates the transponder and also receives the information from the tag. The transponder is attached on the object which is desired to be identified. There are two types of transponders, passive and active. Passive tags have no energy sources; they use energy which is scavenged from the reader. Active tags, on the contrary, have their own batteries [1].

There are many applications for fault detection and condition monitoring in industry. During the process, critical parameters such as strain, temperature, humidity etc. should be monitored with the sensors. The RFID tags which include integrated circuit have high cost values and they are not feasible to use them for small dimensional products. In that case development of chipless RFID sensors play critical role for low-cost, efficient tagging and sensing applications [2].

A. Rida et al. [3] informed that barcodes are mostly used data identification products which are nonprogrammable and have short read range with using limited data. They have many limitations in operation such as human intervention need with less data handling capability. Against these limitations, lowcost benefit makes them very feasible to use in many applications. There is a pressing need to remove the
Application Specified Integrated Circuit (ASIC) from the RFID communication to make it reasonable for replacing barcodes with the tags.

\section{CLASSIFICATION OF RFID SENSORS}

Sensors can be classified with many captions related with their usage area, included component or energizing type etc. According to RFID area, they can be classified as active sensors and passive sensors as shown in Fig. 1. As discussed in introduction section, it is related with their energy sources. If they use energy which is scavenged from the reader, it is passive. Active sensors, on the contrary, have their own batteries.

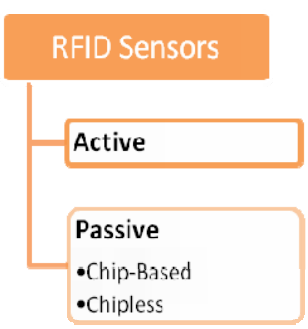

Fig. 1: Classification of RFID Sensors

\section{A. Active RFID Sensors}

Active sensors are not chipless components. The important part of the tag is the microcontroller, which has the biggest role with connecting the sensors to the RF protocol. In Fig. 2, the block diagram of an active sensor tag is illustrated. The sensors are combined to the microcontroller through the sensor bus and these sensors can be a strain sensor, temperature sensor, gas sensor, or any sensor that senses the change in any physical parameters.

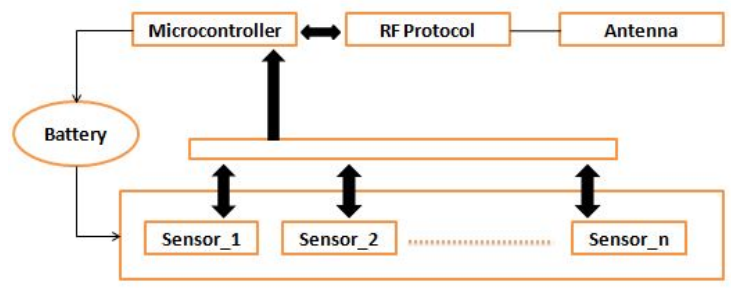

Fig. 2: Block Diagram of Active Sensors 


\section{B. Passive Chipless RFID Sensors}

The chipless RFID sensor affords identification and senses a number of environmental parameters without having an active sensor in the circuitry. The essential difference of these sensors compared with chip-based passive sensors is the nonappearance of a silicon chip in the circuit. The needed energy is scavenged from the reader side. The chipless RFID sensors have more advantages than the traditional sensors because of their stability, longer life and lower cost properties. Chipless tags can be printable on metals and bottles due to compactness property.

J. Dowling et al. [4] stated that the communication range of traditional passive RFID systems is of the order of 3-6 meters, with related to differing surfaces or materials. The field of chipless RFID systems increases up to 20 meter. Types of chipless sensors are shown in Fig. 3.

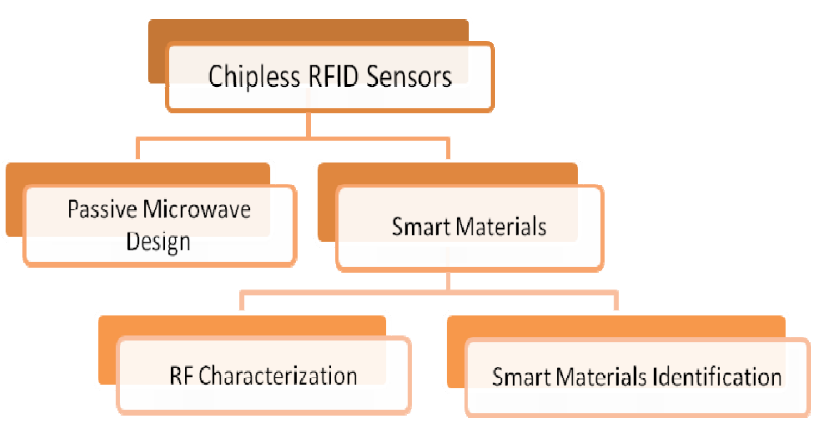

Fig. 3: Classification of Chipless RFID Sensors

As an example of development steps, wireless and batteryfree platform, called Wireless Identification and Sensing Platform (WISP) which is patented by Intel can be given. This system includes a foil strain gage additionally printed Wheatstone bridge and amplifier. The strain measurement utilizing the WISP technology is repeatable and precise as classical wired strain gages and extensometers. [5]

\section{SMART SENSING MATERIALS}

The essential feature of the suggested chipless RFID sensor is "smart materials," also can be named as sensing materials. These materials show huge physical and/or chemical reactions to external effects, such as temperature, pressure, strain, and electric fields, and hence are suitable for monitoring applications. They have great potential to implement RFID devices into monitoring, tracking or recognising applications.

Smart materials which will be used as a sensor needs a characterisation process for improving the design parameters of the material related with the application area. There are different ways of characterisation as stated below.

Some of the materials such as Graphene and Silver can be used for multiple purposes. As an example Graphene material is used to print an antenna for communication, and at the same time, they can also be used as a strain sensor as seen in Fig. 4.

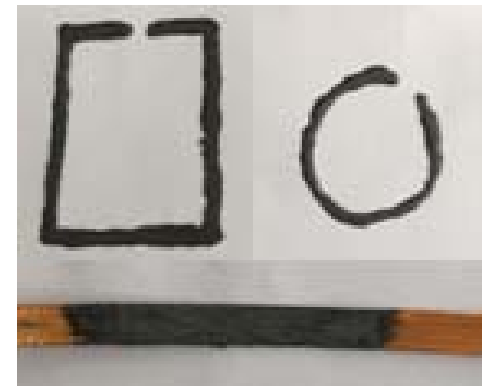

Fig. 4: Creation of an Antenna and Sensor

X-ray Diffraction (XRD) and scanning electron microscopy are mostly prefered methods to analyze the microstructure of material. Atomic Force Microscopy (AFM) is interested in the surface design of the material. Additionally, optical, electrical and thermal properties should also be characterised. DC conductivity, thermal stability and backscatter efficiency are the other critical parameters which should be considered during smart material design.

\section{A. Temperature Sensing Materials}

Temperature is critical parameter for condition monitoring applications. Breakage failures, undesired working conditions and damages can be detected or monitored with the help of temperature parameter.

J. Virtanen and A. Z. Elsherbeni [6] described that pure water can be a temperature sensing material. The use of distilled water supports temperature sensing ranging from $0{ }^{\circ} \mathrm{C}$ up to $100{ }^{\circ} \mathrm{C}$. It is also a cost-effective and environmental friendly sensing type. The sensor can be used to monitor both air and other environmental part temperatures.

As a smart material type, Phenanthrene $\left(\mathrm{C}_{14} \mathrm{H}_{10}\right)$ is a sublimation item from the hydrocarbon group, which transfer directly from the solid to the gas phase without giving a symptom of any intermediate phase. They started to be used in optics and printed electronics.

Ionic plastic crystal is another material which is a kind of plastic crystal with powerless contacted molecules that contain arranged degrees of freedom. They are in intermediate phase between liquid and solid phases. In that case they have really effective solutions for real-time temperature monitoring.

\section{B. Strain Sensing Materials}

Strain is another critical parameter, especially monitoring the breakage failures for constructions, structures, power transmission belts etc. T. T. Thai et al. [7] informed that strain sensing is needed for architecture safety such as bridges and buildings to prevent unexpected collapses. In the same way strain sensing allows the vibration monitoring, enormous loading and undesired crack detection to ignore breakage failures with early detection. In medical uses strain sensing initiate bone implantable sensors and bone fracture monitoring. In aerospace, strain play key role on sensitive operations for aircrafts, including the status of wings and blades, and also fatigue observation on the body of an aircraft.

The connection between stress and strain is one of the most elementary ideas from the study of the mechanics of materials. 
When a force is applied on any surface, the surface distorts. In the common case, this deformation is called strain. In the applications, "strain" term means deformation per unit length or partial change in length. [8]

Strain sensors have been widely developed with using various techniques. The most traditional sensors are strain gages (Fig. 5). These types of sensors make a calculation of strain with the effect of each of strain wires, or gages. On the bleeding edge, several materials such as Nickel-Titanium (Nitinol) alloy, Glass microfiber-reinforced Polytetrafluoroethylene (PTFE), Polyester-based stretchable fabric can be used as strain sensors for fault detection applications. PTFE-based material sensor has wide resonance frequency change property because of its big dielectric constant under temperature fluctuation.

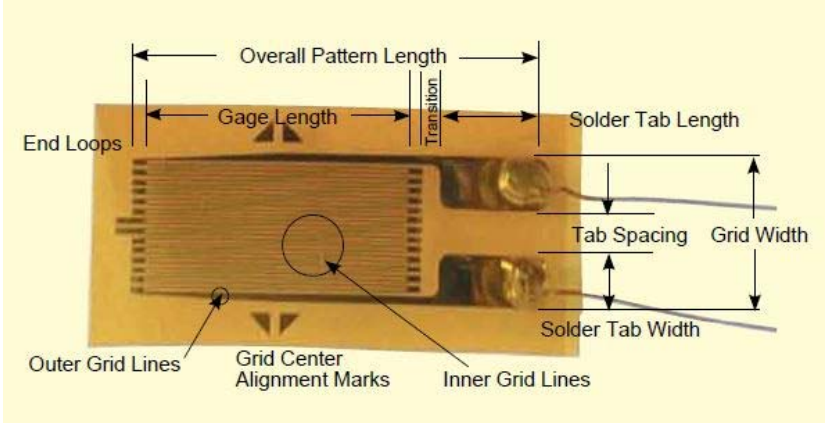

Fig. 5: Critical Parameters of Strain gage [8]

J. P. Burman explained [9] a unique design of a low cost flex sensor which include aluminium foil, cleaning pad, tape, graphite powder and etc. The working principle of the sensor based on the bending movement. The resistance value changes regarding to bending angle. For instance in $180^{\circ}$ condition, resistance increases to maximum value. When the sensor is bent with $90^{\circ}$, its resistance value decreases.

In our research, some experiments are performed with graphene powder material including different adhesive types as shown in Fig. 6. Resistance values are recorded in different bending angles.

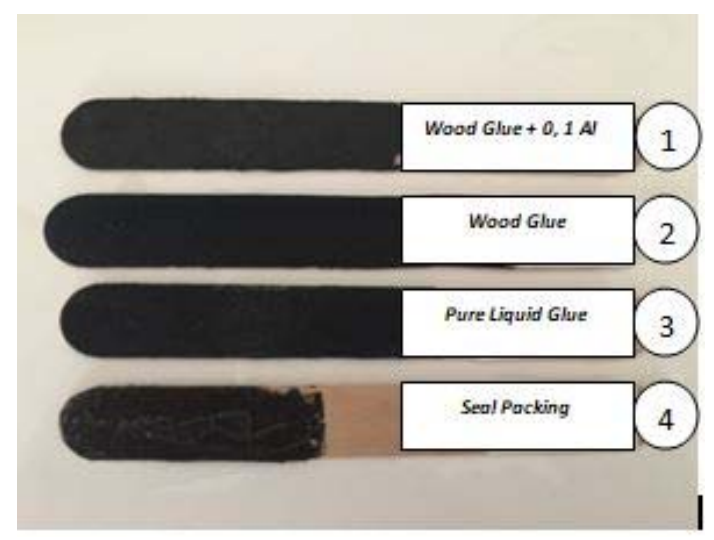

Fig. 6: Strain Performance with Different Materials
As a conclusion when we read the resistance values from the sensor sticks, it increases up to $5 \mathrm{~K} \Omega$.Various adhesive materials perform different electrical conductivity as seen on Table 1.

TABLE I. Resistance VAlues of GRAPHENE POWDERS

\begin{tabular}{|c|c|c|}
\hline Sensor Sample & $\begin{array}{c}\text { Minimum Resistance } \\
(\mathbf{\Omega})\end{array}$ & $\begin{array}{c}\text { Maximum Resistance } \\
(\mathbf{\Omega})\end{array}$ \\
\hline $\begin{array}{c}\text { S_1 } \\
\text { (liquid glue + 0.1 Al) }\end{array}$ & $0.7 \mathrm{~K}$ & $5 \mathrm{~K}$ \\
\hline S_2 (wood glue) & $0.34 \mathrm{~K}$ & $1,6 \mathrm{~K}$ \\
\hline S_3 (pure liquid glue) & $0.4 \mathrm{~K}$ & $4 \mathrm{~K}$ \\
\hline S_4 (seal packing) & No value & No value \\
\hline
\end{tabular}

\section{USE IN CONDITION MONITORING}

Regarding to machinery health monitoring, "Diagnostics" define that detecting fault on a machine and determine cause and effect. Differently "Prognostics" help us to make a comment on the behaviour of any machine (or any product) and evaluate the potential failures on performance before fault occurs.

RFID sensors are widely used for many functions such as monitoring, positioning, tracking, recognition (Fig. 7). Variously bearing fault detection, crack detection, vibration analysis, transmission faults, serial production layers monitoring examples are the main usage areas of fault detection and condition monitoring applications.

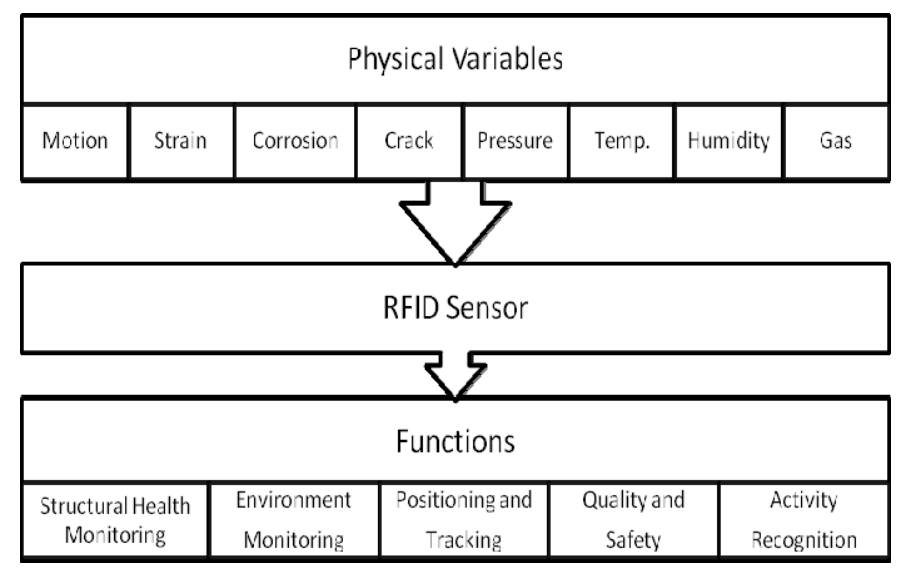

Fig. 7: Usage of RFID Sensor

As Z. Meng and Z. Li stated [10]; there are some technical challenges when silicon chips are removed from the communication. The first one is sophisticated calibration step for accurate measurement. Electromagnetic sensors are affected by environmental effects, therefore calibration is needed. They perform real-time measurement; in that case data flow is affected by noise as a second challenge. Undesired electromagnetic coupling between tags, confidentiality and data protection are the other challenges that should be overcome. 


\section{CONCLUSION}

The excited development of chipless RFID sensors will create huge opportunities for economic and compact design applications in variety of areas. They will reform the identification market of low cost product tagging and sensing, especially for condition monitoring applications.

This paper overviewed the chipless RFID sensors with focusing on critical materials for strain and temperature sensing to contribute the IoT (Internet of Technology) that communicates each and every physical object to transfer of information and condition in today's high technology era.

\section{REFERENCES}

[1] K. Finkenzeller, "RFID Handbook, fundamentals and applications in contactless smart cards, Radio Frequency Identification and Near-Field Communication", John Wiley \& Sons, Ltd, UK, 2010

[2] N. C. Karmakar, E. M. Amin, J. K. Saha, "Chipless RFID sensors", John Wiley \& Sons, Inc, New Jersey, 2016

[3] A. Rida, L. Yang, M. Tentzeris, "RFID-Enabled sensor design and applications" Artech House, 2010

[4] J. Dowling, M. M. Tentzeris, N. Beckett, "RFID-enabled temperature sensing devices: A Major step forward for energy efficiency in home and industrial applications" IEEE MTT-S International Microwave Workshop on Wireless Sensing, Local Positioning, and RFID (IMWS 2009 - Croatia)

[5] F. Gasco et al., "Wireless strain measurement for structural testing and health monitoring of carbon fiber composites" Composites, Part A, Applied Science and Manufacturing, September 20100

[6] J. Virtanen, A. Z. Elsherbeni, "Temperature sensor tag for passive UHF RFID systems" 978-1-4244-8064-7/11/\$26.00 2011 IEEE

[7] T. T. Thai, H. Aubert, P. Pons, M. M. Tentzeris, R. Plana, "Design of a highly sensitive wireless passive RF strain transducer" 978-1-61284-757-3/11/\$26.00 2011 IEEE

[8] Application Note 290-1 "Practical strain gage measurements", Omega, Reproduced with Permission, Courtesy of Agilent Technologies Inc., 1999

[9] J. P. Burman, "Novel design of low cost flex sensor for automatic controlling of robotic car" International Journal of Advanced Research in Electronics and Communication Engineering (IJARECE - March 2015), Volume 4, Issue 3,

[10] Z. Meng, Z. Li, "RFID Tag as a sensor - A Review on the innovative designs and applications" Measurement Science Review, 16, (2016), No. 6, 305-315

[11] Gates Industrial Company, Test laboratory internal training documents, 2017, Izmir

[12] S. Ozdemir, "ME587 Microcontroller Embedded Systems" Lecture Notes, IZTECH, 2017, Izmir 